\title{
Effect of modification to tongue and basic circle diameter on vibration in a double-suction centrifugal pump
}

\author{
Song Chunchun ${ }^{1, a}$, Su Yongsheng ${ }^{2, b}$ \\ ${ }^{1}$ East China University of Science and Technology, Shanghai,China; \\ achunchunsong@163.com,
}

Keywords: Double-suction centrifugal pump; Velocity fluctuation; FLUENT; Numerical simulation

\begin{abstract}
In order to obtain the effect of modification to the tongue gap on vibration when medium is oil in a double-suction pump.The three-dimensional unsteady numerial method was applied to simulate the flow with different volute tongue and base circle diameter by using realizable $\kappa-\varepsilon$ turbulence model. The distributions of pressure and velocity in the pump were obtained with different gaps and velocity hybrid were analyzed by using the concept of velocity fluctuation. The analysis results gave the best tongue angle and the best base circle diameter when oil as working medium which make the lowest vibration. And indicated that the measure of increasing the base circle to reduce the pump vibration is better than the measure of increasing the tongue angel.
\end{abstract}

\section{Introduction}

Double-suction centrifugal pump are widely used in mines, factories, urban water supply and drainage , irrigation engineering, and other fields due to its high flow rate etc. It is one of the most important hydraulic tools. However, as a rotary fluid machinery, some mechanical and hydrodynamics problems always induce large vibration during the operation. One of the most important reasons is pressure fluctuation and velocity fluctuation which caused by the interaction between impeller and volute[1], which has been the key issue in the field of hydraulic machinery.

A double-suction centrifugal pump produced huge vibration and noises when operating in a factory especially when the medium is oil which would seriously threat the safe operation of the unit. So the pump need to be modified urgently to reduce its vibration. Many studies have shown that the pressure fluctuation, which is caused by rotor-stator interaction, is one of the most important factors that affect pump running stability. They also conclude that the change of radial gap between impeller and volute tongue has huge influence on pressure pulsation characteristics of centrifugal pump[2,3]. To study the influence on pressure fluctuation, most of researchers change the radial gap between impeller and volute tongue by changing the volute tongue angle and cutting back of impellers[4,5]. However, the performance parameter would be changed when cutting back of the impellers, especially the pump head. In addition, most of this papers obtained internal flow field when pump working medium is water. Hence, this paper took the double-suction centrifugal pump as the model pump and oil as the working medium, and established different models by changing the volute tongue angle and basic circle diameter under the condition of not changing the parameter of the impeller. For each model, numerical simulation was conducted by using CFD. In order to study the effect of the radical gap between impeller and tongue on velocity fluctuation of pump and find the best model which has the lowest vibration, the concept of velocity fluctuation rate was proposed and applied in this paper.

\section{Numerical Model}

The 3-dimension model pump is established by PRO/E software according to the 2D drawing. In addition, the inlet and outlet of the pump has been extended properly in order to increase the stability of the calculation. The main characteristics of the pump at rated point are as follows.

Pump flow rate $Q=874 \mathrm{~m}^{3} / \mathrm{h}$, Outlet diameter of impeller $d_{2}=457 \mathrm{~mm}$, Nominal rotational speed $n=2970 \mathrm{r} / \mathrm{min}$, Pump head $H=250 \mathrm{~m}$, Number of blade $z=4$, Inlet diameter of impeller 
$d_{1}=380 \mathrm{~mm}$.

In order to study the impact of different tongue angels on the rotor-stator interaction, this paper selected 5 kinds of different angels. As shown in Fig.1, they are $1^{\circ} 、 5^{\circ} 、 9^{\circ} 、 13^{\circ} 、 17^{\circ}$ respectively . In addition, the gap rate $S$ which is the ratio of the gap and the outlet radius of impeller, is defined to analyze the change of volute tongue-impeller gaps quantificationally. And the equation is as below:

$$
S=\left(R_{3}-R_{2}\right) / R_{2}
$$

Where, $R_{2}$ is the outlet radius of impeller, $R_{3}$ is the distance from the head of tongue and the center of impeller. So the S of different tongue angels are $4.16 \%, 4.3 \%, 4.4 \% 、 4.55 \%, 4.7 \%$ respectively. Besides, 5 kinds of different basic circle diameter are also selected in order to study the impact of different base circle diameter on the rotor-stator interaction in this paper. They are $457 \mathrm{~mm} 、 461 \mathrm{~mm} 、 466 \mathrm{~mm} 、 471 \mathrm{~mm} 、 475 \mathrm{~mm}$ respectively and as shown in Fig.2. The corresponding gap rate $\mathrm{S}=4.16 \%, 5.0 \% 、 6.1 \% 、 7.0 \% 、 8.1 \%$.

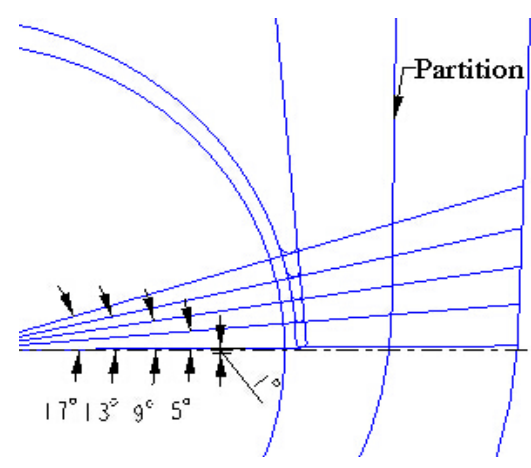

Fig.1 Volute diagram of different volute tongue

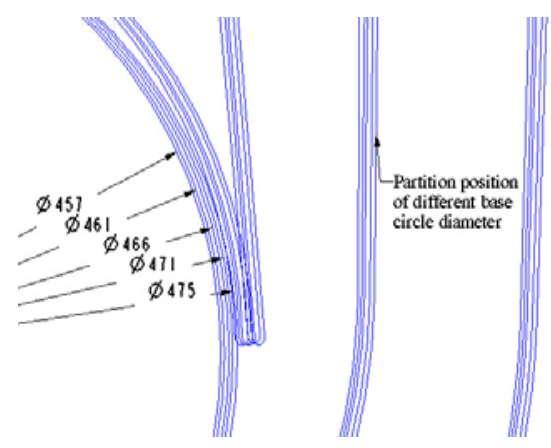

Fig.2 Volute diagram of different base circle diameter

The geometry discretization is made to keep the balance between calculation time and the accuracy order of the simulation of the flow structure. Considering the complexity of the double-suction centrifugal pump, unstructured tetrahedral cells are generated to define the impeller and volute, while structure hexahedral cells are used to define the inlet and outlet zones in order to save the simulation time. In the volute, a mesh refinement zone is taken near the tongue and the splitter. There are in total of 1.9 million cells in the computational domain. The view of the generated grid is shown in Fig.3. Same gridding strategy is utilized to grid the models with different radical gap. The difference between them is just the cell number.
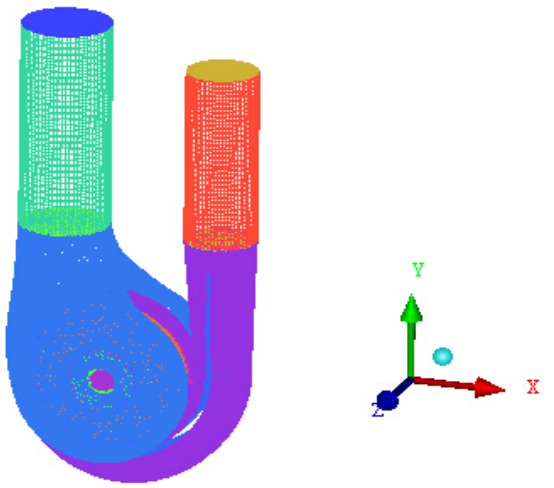

Fig.3 Meshes in whole calculating region

\section{Numerical Computation Method}

Governing Equation. The phenomenon of flow separation and secondary flow is easy to appear in double-suction centrifugal pump because of its high rotation speed. So Realizable $\kappa-\varepsilon$ is used to simulation due to its good performance for rotating flow, the flow separation and the complex flow. 
The control equations are as follows:

Continuity equation:

$$
\frac{\partial \rho}{\partial t}+\frac{\partial}{x_{i}}\left(\rho u_{i}\right)=0
$$

Momentum equation:

$$
\frac{\partial}{\partial t}\left(\rho u_{i}\right)+\frac{\partial}{\partial x_{j}}\left(\rho u_{i} u_{j}\right)=-\frac{\partial P}{\partial x_{i}}+\frac{\partial}{\partial x_{j}}\left(\mu \frac{\partial u_{i}}{\partial x_{j}}-\overline{\rho u_{i}^{\prime} u_{j}^{\prime}}\right)
$$

Where, $\rho$ is density, $u_{i}$ 、 $u_{j}$ is velocity vector, $\mathrm{P}$ is pressure, t is time, $\mu$ is coefficient of viscosity. Realizable $\kappa-\varepsilon$ is used to make the Reynolds equation closed,

$$
\begin{gathered}
\rho \frac{D k}{D t}=\frac{\partial}{\partial x_{i}}\left[\left(u+\frac{u_{t}}{\sigma_{k}}\right) \frac{\partial k}{\partial x_{i}}\right]+G_{k}+G_{b}-\rho \varepsilon-Y_{m} \\
\rho \frac{D \varepsilon}{D t}=\frac{\partial}{\partial x_{j}}\left[\left(u+\frac{u_{t}}{\sigma}\right) \frac{\partial \varepsilon}{\partial x_{j}}\right]+\rho C_{1} S \varepsilon+\rho C_{2} \frac{\varepsilon^{2}}{k+\sqrt{v \varepsilon}}+C_{\varepsilon 1} \frac{\varepsilon}{k} C_{\varepsilon 3} G_{b}
\end{gathered}
$$

Where, $\eta=S k / \varepsilon, \mu_{t}=\rho C_{\mu} \frac{k^{2}}{\varepsilon}, C_{\mu}=\frac{1}{A_{0}+A_{S} \frac{U^{*} k}{\varepsilon}}, G_{b}$ is the turbulent kinetic energy generation related to buoyancy, $G_{k}$ is the turbulent kinetic energy generation related to the average velocity gradient, $Y_{m}$ is the effect of compressible turbulent pulsating expanding to the total dissipation rate. The model constants are as below:

$$
C_{\varepsilon 1}=1.44, C_{2}=1.9, \sigma_{k}=1.0, \sigma_{\varepsilon}=1.2
$$

Boundary conditions and numerical methods. In this study the whole flow field of the double-suction centrifugal pump is simulated by using FLUENT software. Because of rotating impeller, multiple reference frame model was employed to the interaction of impeller and volute, so it can simplify the complex unsteady field into the steady flow field of a fixed rotating impeller at a particular location. Turbulence was simulated by means of a realizable $\kappa-\varepsilon$ model, and standard wall functions were used to calculate boundary layer variables. The pressure-velocity coupling was established by means of the SIMPLEC algorithm. Second order upwind discretizations were used for the convection terms and central difference schemes the diffusion terms. Owe relaxation factor are the default values. As boundary conditions, a velocity was considered at the inlet and out-flow at the outlet dependent on the flow rate, and no slip boundary condition was used to handle the wall of pump.

\section{Result Analysis}

Pressure Analysis. Fig. 4 and Fig. 5 shows the pressure distribution of the section at $Z=12 \mathrm{~mm}$ and impeller surface when working medium is oil(Section $Z=0$ is the symmetry of double-suction centrifugal pump). From Fig. 4 we can see that the pressure distribution along the two parts of volute is similar, and they are symmetrical to each other. That is caused by the interaction between impeller and the two parts of symmetrical volute. In addition, in this picture we can also find that high pressure existed in local near the around tongue and head of splitter. That means that flow is accelerated near this location due to blockage effect. The analysis above shows that the structure of volute has a great influence on flow of the outlet of impeller. Fig. 5 shows that the state pressure at the inlet of impeller is minimum and increases with the increase of the radius until the maximum state pressure turned up at the outlet. In addition, the state pressure of pressure surface is obvious higher than the suction surface's at the same radius. What's more, there is low pressure area at the impeller inlet, whose pressure value was negative and the cavitation is most likely to occur. 


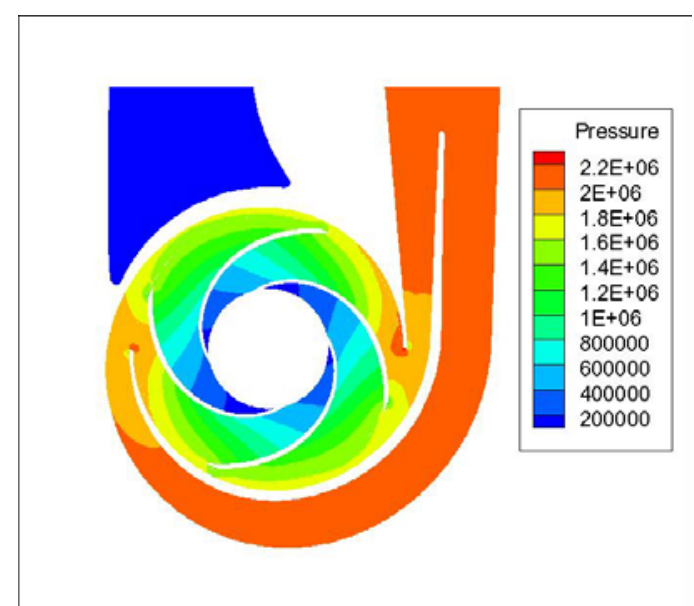

Fig.4 Static pressure contour on the $\mathrm{Z}=12 \mathrm{~mm}$ section

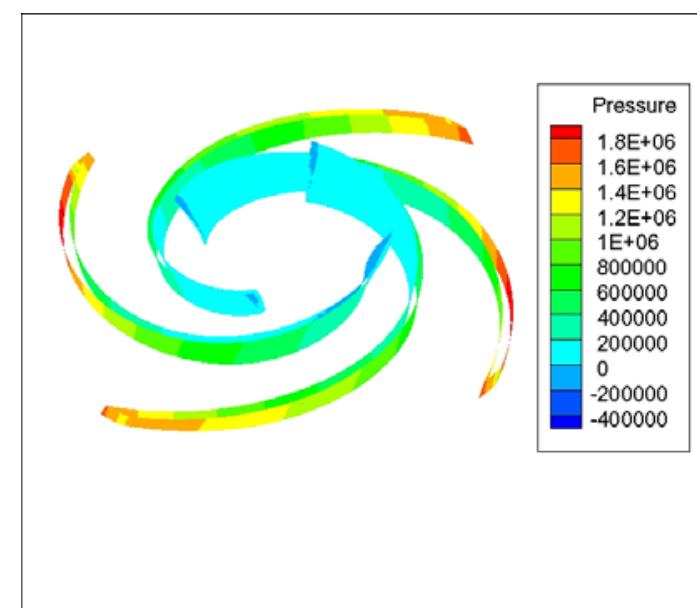

Fig.5 Static pressure contour on the surface of impeller

Velocity fluctuation analysis of different tongue and base circle diameter. The non-uniform distribution of velocity at impeller exist is key reason of centrifugal pump vibration. In order to make a further study of the circumferential distribution of velocity on different diameter in the volute, coefficient of velocity fluctuation $C_{v}$ is introduced, the equation is as below:

$$
C_{v}=\left(v_{\max }-v_{\min }\right) / \bar{v}
$$

Where, $v_{\max }$ is maximum speed of a circumference on the $\mathrm{Z}=12 \mathrm{~mm}$ section in the volute. $v_{\min }$ is minimum speed of a circumference on the $Z=12 \mathrm{~mm}$ section in the volute. $\bar{v}$ is average speed of a circumference on the $\mathrm{Z}=12 \mathrm{~mm}$ section in the volute. The bigger $C_{v}$ means the bigger velocity gradient of the pump along the circumference, the bigger the velocity fluctuation, the more nonuniform the velocity distribution, the bigger vibration the pump will be.

Velocity fluctuation of different tongue angle on different circumference in volute of the centrifugal pump was showed in Fig.7(ge- $1^{\circ}$ means the model whose tongue degree is $1^{\mathrm{o}}$, and others). It can be observed that the changes of velocity fluctuation in the volute at different radius, so that we can analyze mixture of velocity in the volute of the pump . Fig.7 shows that the velocity fluctuation decrease gradually as radius increasing in the volute. That means the flow mix evenly step by step from the impeller exist to the volute casing. Fig.7 also shows that the magnitude of velocity fluctuation reduction increases firstly, and then decreases with increase of the tongue angle. That depicts that there is a optimum tongue angle to make the best flow field. Which the flow in the volute will mix most evenly and the vibrations is smallest. From the Fig.7, we can see the tongue angle is 13 o.

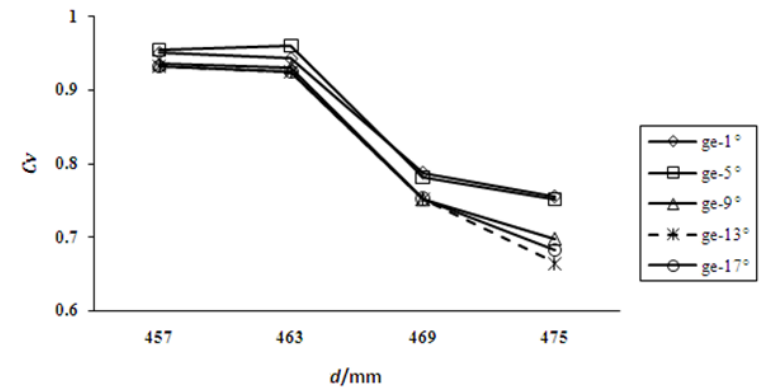

Fig.7 Velocity fluctuations in different circumference of pump in different volute tongue when medium is oil

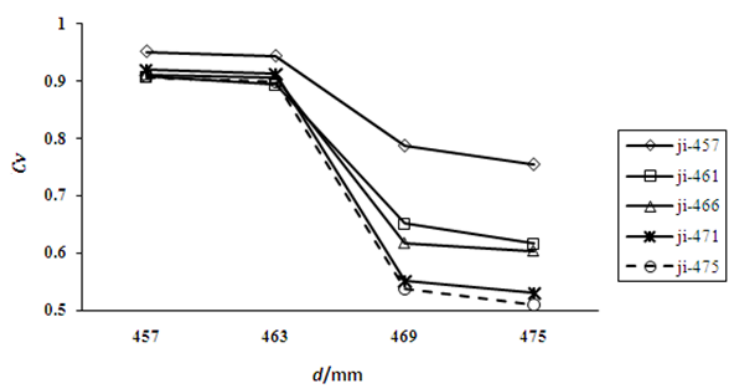

Fig.8 Velocity fluctuations in different circumference of pump in different base circle diameter when medium is oil

Similarly, Fig.8 shows the velocity fluctuation of different basic circle diameter on different circumference in the volute of centrifugal pump (ji-457 means the model which basic circle diameter is $457 \mathrm{~mm}$, and others). It can be figured out that the velocity fluctuation decrease as the diameter 
increasing in the volute. And that indicates that the flow mix evenly step by step from the impeller exist to the volute casing. In addition, the velocity fluctuation firstly decrease and then keep constant with increase of the basic circle diameter. It means that there is an optimistic basic circle diameter make inner flow field flow best, which fluid in the volute mix most evenly and the vibration is smallest. Fig. 8 shows the best basic circle diameter is $475 \mathrm{~mm}$.

Fig. 9 shows the comparison of velocity fluctuation in the volute between two different model(one is ge- $13^{\circ}$, the other is ji-475). From Fig.9, we can figure out that the magnitude of velocity fluctuation reduction in the model ji- 475 is bigger than the model ge- $13^{\circ}$. So we can conclude that the measure of increasing basic circle diameter is more effective than that of increasing tongue angle.

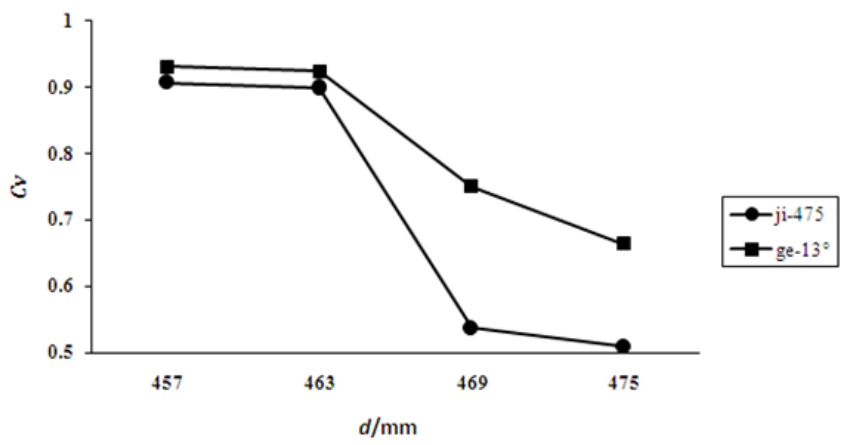

Fig.9 Velocity fluctuations in different circumference of pump at the best tongue angle and base circle diameter when medium is oil

\section{Conclusion}

In this study, steady calculation methods have been applied to obtain the information of inner flow field in the pump when working medium is oil. Based on the calculation results, the conclusions are as follows:

(1) The structure of volute has a great effect on the flow of impeller exist. Volute tongue and splitter has a great effect on pressure distribution and velocity distribution in the inner flow field of centrifugal pump.

(2) The velocity fluctuation near the volute tongue greatly decrease by increasing the tongue angle and basic circle diameter of the pump properly. We can conclude that the best tongue angle is $13^{\circ}$ and the best base circle diameter is $475 \mathrm{~mm}$ when medium is oil. With this two methods, the vibration of the pump is reduced greatly.

(3) The method of increasing basic circle diameter of pump is better than that of increasing tongue angle. To achieve the best performance and the smallest vibration of the double-suction centrifugal pump when working in oil medium, we can choose the bigger basic circle diameter which within a reasonable range.

\section{References}

[1] Zhang, M., \& Tsukamoto, H. (2005). Unsteady hydrodynamic forces due to rotor-stator interaction on a diffuser pump with identical number of vanes on the impeller and diffuser. Journal of fluids engineering, 127(4), 743-751.

[2] González, J., Fernández, J., Blanco, E., \& Santolaria, C. (2002). Numerical simulation of the dynamic effects due to impeller-volute interaction in a centrifugal pump. Journal of Fluids Engineering, 124(2), 348-355.

[3] Cong, G. H., \& Wang, F. J. (2008). Numerical investigation of unsteady pressure fluctuations near volute tongue in a double-suction centrifugal pump.Transaction of the Chinese Society of Agricultural Machinery, 39(6), 60-63. 
[4] Barrio, R., Blanco, E., Parrondo, J., GonzÃĄlez, J., \& FernÃĄndez, J. (2008). The effect of impeller cutback on the fluid-dynamic pulsations and load at the blade-passing frequency in a centrifugal pump. Journal of Fluids Engineering, 130(11), 111102.

[5] Dong, R., Chu, S., \& Katz, J. (1997). Effect of modification to tongue and impeller geometry on unsteady flow, pressure fluctuations, and noise in a centrifugal pump. Journal of Turbomachinery, 119(3), 506-515 